\title{
The Effects of the Presence of Multiple Conduction Velocities in the Analysis of Electrically-Evoked Compound Action Potentials (eCAPs)
}

\author{
J Taylor, S. Sadrafshari, N. Donaldson, N Granger, J Prager and B Metcalfe
}

\begin{abstract}
New methods for the analysis of electricallyevoked compound action potentials (eCAPs) are described. Mammalian nerves tend to have broad multi-modal distributions of fibre diameters, which translates into a spread of conduction velocities. The method of velocity selective recording (VSR) is unable to distinguish between this spectral spread and the transfer function of the system. The concept of the velocity impulse function (VIF) is introduced as a tool to differentiate between these signal and system attributes. The new methods enable separate estimates of velocity spectral broadening and signal-to-noise ratio (SNR) to be obtained.
\end{abstract}

\section{INTRODUCTION}

The development of neural interfaces for recording peripheral nerve activity is a rapidly growing area of research with the potential to provide the necessary motor, sensory, or autonomic control information required in many neuroprosthetic applications [1], [2]. Velocity selective recording (VSR) is a technique that allows information to be extracted from an intact nerve with a recording set-up that does not in general allow action potentials (APs) from single fibres to be seen as spikes [3], [4]. This is usually the case when nerve cuffs are employed as interfaces since the signalto-noise ratio (SNR) tends to be low [5]. The principle of VSR has mostly been developed using single fibre action potential (SFAP) models. VSR has been applied to the analysis of recorded, electrically-evoked compound action potentials (eCAPs) based on the fundamental assumption that these are simply summed SFAPs that are (a) equal in velocity and (b) coincident in time, or nearly so. In reality neither of these assumptions is valid. From the time of Erlanger and Gasser, conduction velocity (CV) distributions from mammalian nerves with broad multi-modal distributions have been recorded (and backed up by histological measurements of fibre diameter) and so assumption (a) in particular seems to be highly questionable. It is to be expected therefore that this distribution of conduction velocities/fibre diameters will translate into a different velocity spectrum from that obtained for a single SFAP using VSR. Since VSR systems have their own spectral properties (essentially a form of bandpass filter in the velocity domain), it is impossible to determine whether the recorded velocity spectrum is a signal or system attribute (or both). This makes the global application of 'useful'

J Taylor, S Sadrafshari and B Metcalfe are with the Department of Electronic and Electrical Engineering, University of Bath, Bath UK, BA2 7AY UK.

N. Donaldson is with the Department of Medical Physics and Bioengineering, University College London, London WC1, UK.

N. Granger and J. Prager are with The Royal Veterinary College, familiar measures such as velocity $Q\left(Q_{v}-\right.$ by analogy to resonant systems) invalid, at least, in the simple form described in [4].

In this paper the concept of the velocity impulse function (VIF) is introduced to resolve this issue by separating the properties of the VSR system itself from those properties attributable to the incident signal. This approach enables a complete velocity spectrum to be synthesized more rapidly than would be possible using a step-by-step time domain approach (typically about 10 s to compute a single spectrum) using a program such as Matlab.

The main observable effects of the statistical variation in velocity are described. These are (a) to reduce the maximum/minimum amplitude ratio of the bandpass filtered velocity spectrum below the nominal value of $N$, where $N$ is the number of electrodes, and (b) to broaden the velocity spectrum. In addition, the effect of additive white Gaussian noise (AWGN) is noted, which also reduces the maximum/minimum amplitude ratio, but importantly, without broadening the spectrum. This is important because it allows the effects of noise and spectral spreading to be separately estimated and a simple method to implement this is described. The simulated results are supported by a set of measured data obtained from an in vivo experiment using pig vagus nerve.

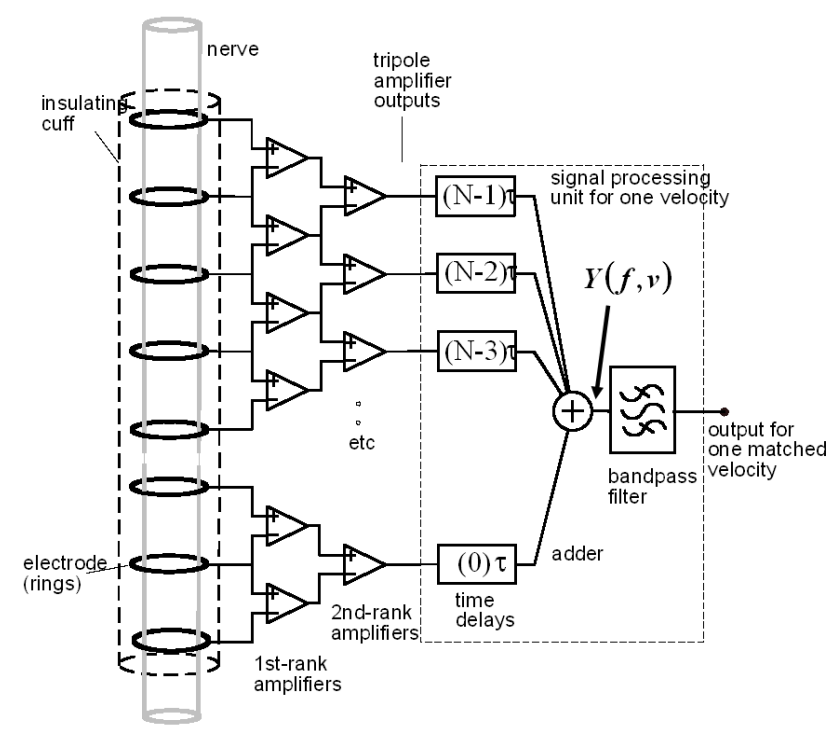

Figure 1. This shows a multielectrode cuff (MEC), on the left of the diagram, connected to a tripolar (double differential) amplifier array. The $N$ tripolar outputs (where $N$ is typically about 10) are delayed and added before being bandpass filtered. 


\section{METHODS}

\section{A. Basic principles}

Figure 1 shows the block diagram of an $N$ channel VSR signal acquisition and processing system [3], [4]. This consists of a multi-electrode cuff (MEC), an amplifier array (shown here in a tripolar or double-differential arrangement),

a delay-and-add stage and finally a bandpass filter (BPF). Analysis of figure 1 results in the following tripolar intrinsic velocity transfer function:

$$
H(f, v)=\left[K 4 . \sin ^{2} \frac{2 \pi f d}{v}\right]\left[\frac{\sin N x}{\sin x} e^{-j(N-1) x}\right]
$$

where:

$$
x=\frac{2 \pi f d}{2}\left(\frac{1}{w}-\frac{1}{W_{0}}\right) \text {, and }
$$

$f$ is the measurement frequency,

$v$ is the velocity ( $v_{0}$ is the matched velocity),

$N$ is the number of electrodes,

$d$ is the inter-electrode spacing, and

$K=R_{e} / R_{a}$, where $R_{e}$ and $R_{a}$ are the extra-axonal and intraaxonal resistances per unit length inside the cuff, respectively.

Equation (1), the tripolar intrinsic velocity transfer function (IVF), is a function of two variables, frequency and velocity. In earlier publications on this subject, in order to obtain a spectrum that is a function of velocity only, the practice at this point has been to define $f$ precisely by cascading the output of the delay-and-add section with a bandpass filter (BPF), so that $f=f_{0}$, where $f_{0}$ is the centre frequency of the BPF. The incident waveform (i.e. an AP) is replaced by a short burst of sinewaves whose characteristics depend on the BPF and not (to a first order of approximation) on the signal itself. The response of the system is therefore largely insensitive to the precise form of the incident waveform [4]. However, since it is customary in practice to use BPFs with quite wide passbands (typically about $10 \%-50 \%$ of $f_{0}$ ), in order to limit the length of the transient response and hence suppress spurious 'image' responses [7], the narrow band approach (i.e. assuming that $f$ $\left.=f_{0}\right)$ is an approximation. In this paper we show that in fact the actual bandwidth of the filter has little significant effect on the overall spectrum and hence the analytic form of the velocity impulse function (VIF) can be considerably simplified. In addition, since the earlier work referred to considered only the spectra of SFAPs, or of ideal eCAPs, it was acceptable to consider just the modulus of $H(v)$. However, as eqn (1) shows, $H$ is in fact a complex function of velocity and so forming eCAPs from SFAPs of different velocities requires complex addition.

\section{B. The velocity impulse function (VIF)}

For the study presented in this paper, beginning with eqn (1), $H$ is simplified to the case of $N$ monopolar electrodes and expressed in normalized form as follows (using angular frequency, where $\omega=2 \pi f$ ):

$$
H(\omega, v)=\frac{\sin N x}{\sin x} e^{-j(N-1) x}
$$

To form the velocity transfer function $H(v), H(\omega, v)$ is cascaded with a BPF of transfer function $G(\omega)$ and bandwidth $\left(\omega_{1} \leq \omega \leq \omega_{2}\right)$, resulting in the following function:

$$
H(v)=\int_{\omega_{1}}^{\omega_{2}} G(\omega) \frac{\sin N x}{\sin x} e^{-j(N-1) x} d \omega
$$

Assuming a simple 'brick-wall' model of the BPF, eqn (3) simplifies to:

$$
H(v) \rightarrow \int_{\omega_{1}}^{\omega_{2}} \frac{\sin N x}{\sin x} e^{-j(N-1) x} d \omega
$$

Eqn (4) is the analytical form of the VIF, i.e. the response of a bandpass filtered, delay-and-add system when driven with a signal containing a single, discrete conduction velocity. However, the integral cannot be evaluated analytically, but can be computed using a program such as Matlab. Figure 2 compares the response of the system considered above, i.e. the response to a single AP calculated in the time domain, with $H(v)$ calculated by direct evaluation of eqn (4), for BPFs with centre frequency $8 \mathrm{kHz}$ and relative bandwidths of 0.1 and 0.5 . There is close agreement between the responses for both values of relative bandwidth, including the modelling of the spurious 'image' response at about $14 \mathrm{~m} / \mathrm{s}$ (note that the image response is more marked for the lower value of relative bandwidth since this corresponds to a longer time domain response [7). Note also that the plots in Fig 2 illustrate an important characteristic of delay-and-add based systems referred to in the introduction, i.e. that the ratio of maximum to minimum amplitudes is $N$ (10 in this case).
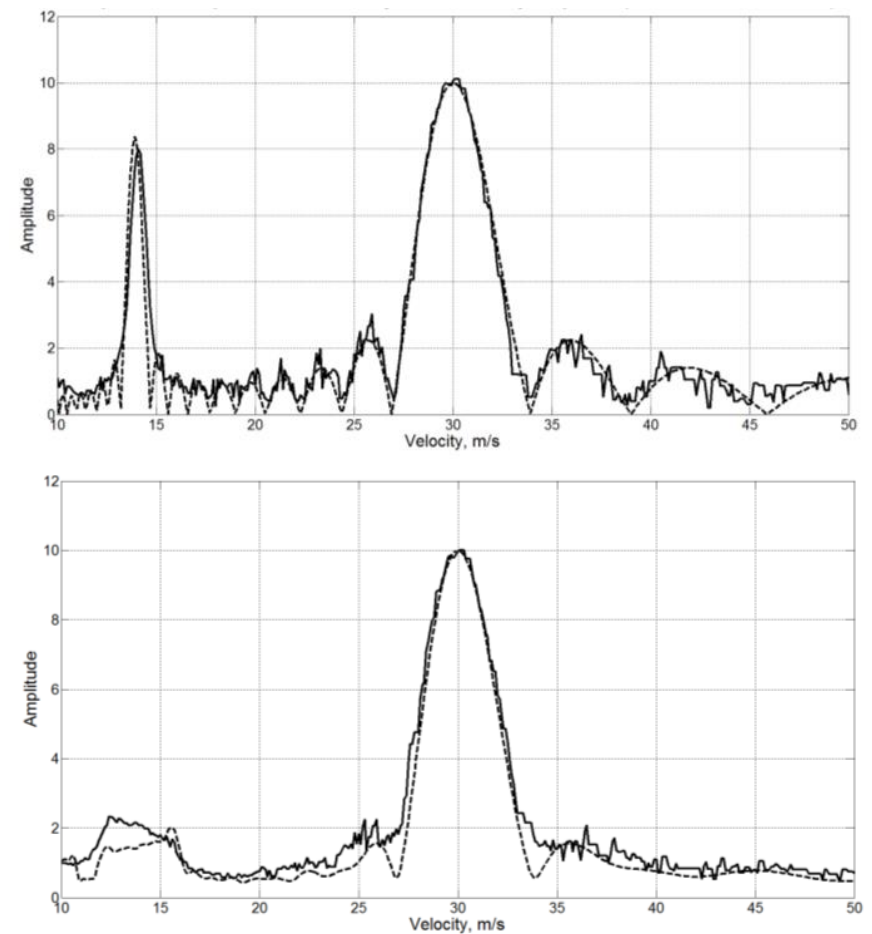

Figure 2. Comparison of time domain calculation of the velocity impulse function (VIF) of an SFAP of velocity $30 \mathrm{~m} / \mathrm{s}$ with the spectrum calculated directly, for relative bandwidths of 0.1 (top) and 0.5 (bottom). Close agreement between the two is shown in both cases. 


\section{Summation of VIFs}

As already noted, in order to combine the spectra of several APs of different velocities to form the spectrum of a CAP, the time-shifted responses are simply summed, before or after delay-and-add and bandpass filtering are applied and before the maximum value is calculated at each velocity. The corresponding method using the VIF is to add the spectra directly, noting that these are complex functions and the modulus is taken only after the compound VIF has been calculated. Note that it is possible to omit the numerical integration step in eqn (4), which has the effect of reducing the bandwidth of the VIF calculation to $1 \mathrm{~Hz}$. In this case although the behaviour around the matched velocity is preserved, accuracy is lost at other velocities. The loss of accuracy away from the matched velocity might in many applications be a price worth paying to achieve the resulting reduction in computational load if the method is to be used as the basis of a statistical spectral analysis of a compound AP.

\section{StATISTICAL ANALYSIS USING VIF}

The VIF method lends itself well to a statistical analysis since the computation required per velocity is much reduced compared to the time domain approach. Figs 3(a) and (b) show the calculation of the summed velocity spectra (VIF) of 1000 APs with random velocities in the range $30 \mathrm{~m} / \mathrm{s} \times(1$ \pm spread), where the parameter 'spread' (standard deviation) is varied as shown from 0 to 0.1 in steps of 0.02 . The bandwidth of the BPF is $50 \%$ of $f_{0}\left(f_{0}=8 \mathrm{kHz}\right)$ and the VIF is calculated using eqn (4) with 1000 steps of numerical integration. The amplitudes of the APs are assumed to be identical and no additive noise was applied at this stage. The main effects of increasing the spread of conduction velocities is to (i) reduce the ratio of maximum to minimum amplitudes to a number less than the nominal SFAP value of $N$ and (ii) to broaden the spectrum. The two subplots use the same data but whereas (a) emphasizes the variation in amplitude, (b) is normalized to show the lateral spread in velocity. Figure 3(c) shows the effect of additive white noise (expressed as SNR) on the velocity spectrum of a single AP. Note that the main effect is to raise the noise floor, effectively reducing the max/min ratio just as in Fig 3(a) for velocity spreading. However, unlike the velocity spread case, there is no spectral broadening. This important observation allows the two effects to be separated without $a$ priori knowledge of the system SNR. Since this parameter, the maximum/minimum ratio of the velocity spectrum at the matched velocity is also a function of additive noise, Fig 4(a) includes plots that show the influence of AWGN. Note the top curve in Fig 4(a) is the case where there is no additive noise (SNR $\rightarrow \infty$ ) and the others indicate the decrease in $\mathrm{max} / \mathrm{min}$ ratio with decreasing signal to noise ratio (SNR) even when there is no velocity spreading.

Figure 4(b) plots the variation in velocity spectral bandwidth as a function of velocity 'spread', for matched velocities of 20, 30, 40 and $60 \mathrm{~m} / \mathrm{s}$. Note that the effect of incident spectral broadening on this parameter is greater for lower matched velocities. In addition, as already noted, this parameter is not affected by additive noise.

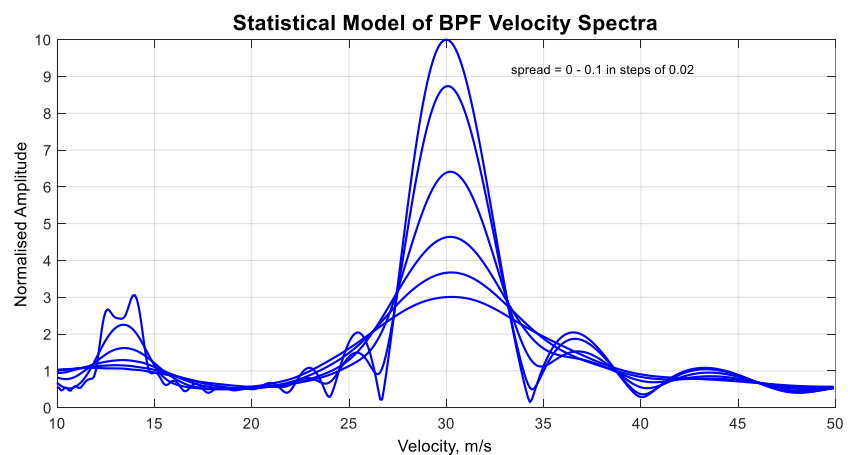

(a)

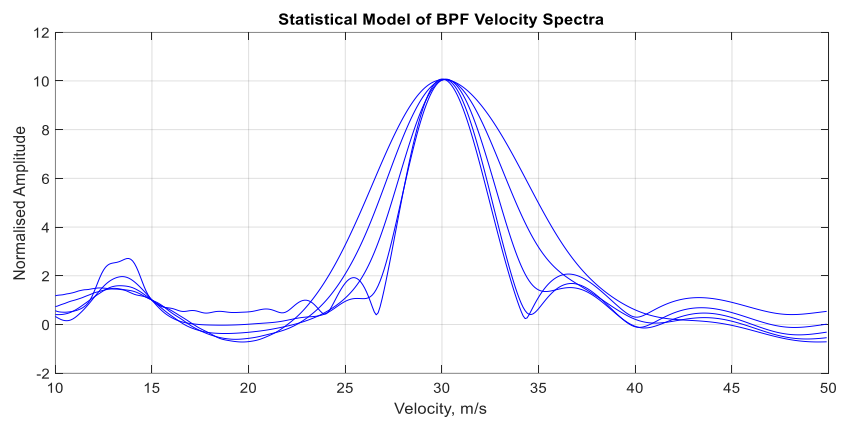

(b)

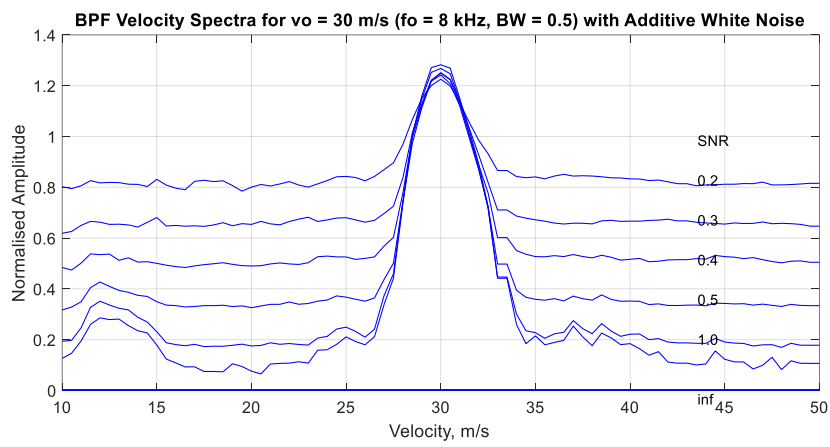

(c)

Figure 3(a), (b) and (c). Calculation of the summed velocity spectra (VIF) of 1000 APs with random velocities in the range $30 \mathrm{~m} / \mathrm{s} \times(1 \pm$ spread $)$, where the parameter spread is varied as shown from 0 to 0.1 . The bandwidth of the BPF is $25 \%$ of $f_{0}\left(f_{0}=8 \mathrm{kHz}\right)$ and the filter transfer function is calculated using 1000 steps of numerical integration. Figure 3(c) shows the effect of additive white noise on a single AP for different values of SNR. Note that although, as in the case of velocity spreading, the max/min ratio decreases with SNR, there is no lateral spreading.

\section{RESULTS}

To illustrate the application of the method, consider a 10 channel VSR system specified as above, to which is applied an input consisting of an eCAP recording of a neural signal whose nominal conduction velocity is $30 \mathrm{~m} / \mathrm{s}$. In order to investigate the velocity spectral purity of this neural signal, the resulting velocity spectrum is compared to the ideal VIF, as shown in the plots in Fig 2. Suppose that the recorded spectrum displays both types of degradation shown in Fig 4, i.e. both spectral broadening and a reduction in the $\mathrm{max} / \mathrm{min}$ 


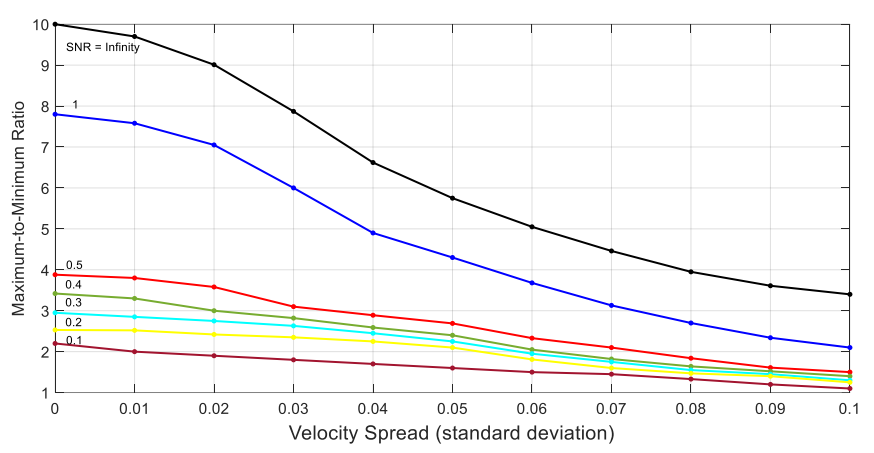

(a)

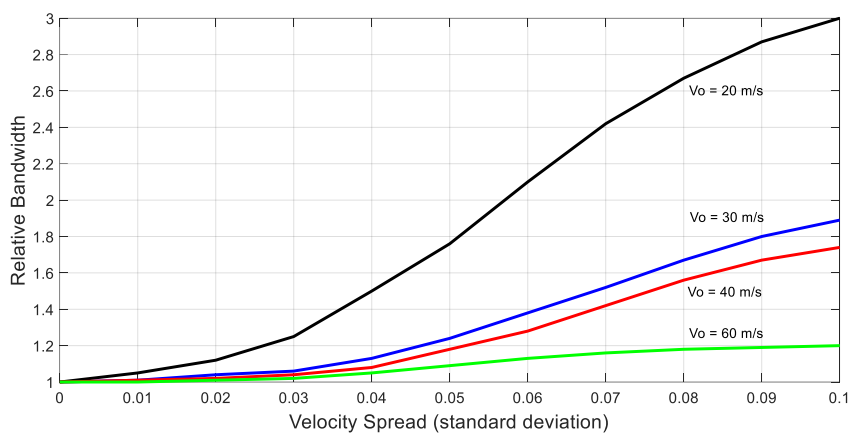

(b)

Figure 4(a) and (b). (a) decrease in $\max / \mathrm{min}$ ratio of the delayed-andsummed output of an $N$-channel VSR system $(\mathrm{N}=10)$ at the matched velocity of $30 \mathrm{~m} / \mathrm{s}$ as a function of the parameter 'spread', i.e. the standard deviation of the velocity distribution. Fig 4(b) shows the increase in velocity spectral bandwidth for the same range of the parameter 'spread' (values from Fig 3(b)). Both sets of values have been normalized to for zero spread.

value below the nominal value of 10 . This suggests both the potential presence of APs with multiple CVs and also noise.

Suppose that the spectral bandwidth has increased by a factor of 1.4 and the $\mathrm{max} / \mathrm{min}$ ratio has reduced to 2.4 . Turning to Fig 6 (b), this corresponds to a velocity spread (standard deviation) of 0.6 (note that this can be determined from the spectral broadening alone since noise does not affect this parameter). Referring finally to Fig 6(a), for a velocity spread of 0.6 and a max/min ratio of 2.4 , an SNR of 0.5 is predicted. These observations suggest the following 3 step procedure:

1. use VSR to estimate the mean of the velocity distribution;

2. compare the recorded velocity profile with the VIF to estimate the spread of the input spectrum (Fig 6(b));

3. estimate the SNR (Fig 6(a)).

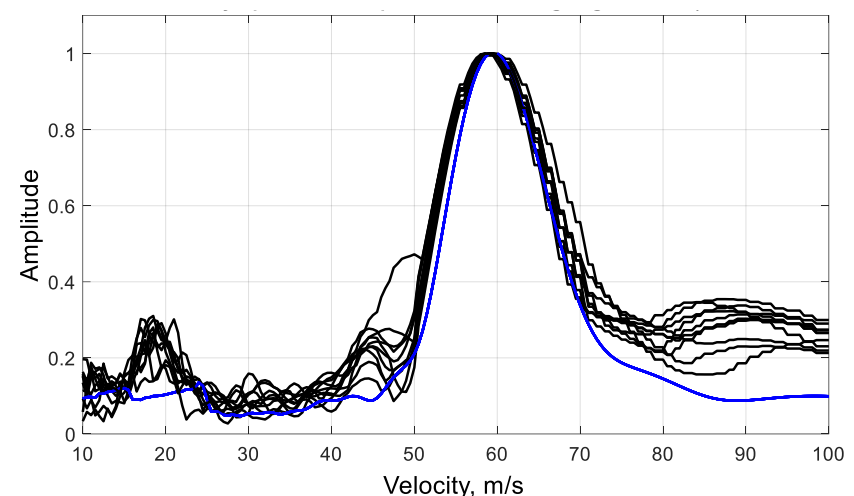

Figure 5. Measured velocity spectra taken from pig vagus nerve using an 11 electrode cuff connected as 10 dipoles. Ten separate runs are shown, each with the same level of electrical stimulation and with the computed VIF shown for comparison (blue trace).

In order to demonstrate the application of the method, a set of measured data is considered, resulting from acute experiments on pig vagus nerve in vivo [5]. The data was obtained using an 11 electrode cuff connected as 10 dipoles. A single Butterworth digital BPF of centre frequency $8 \mathrm{kHz}$ and relative bandwidth 0.5 was connected after the delayand-add stage. The data is shown in Fig 5. Ten separate runs are shown, all with the same level of electrical stimulation. The VIF is shown for reference and although there is very little spectral broadening, suggesting little or no spreading of the incident velocities, the $\mathrm{max} / \mathrm{min}$ ratio is reduced from its ideal level of 10 to about 5 . This suggests the presence of noise and, referring to Fig 5(c), and bearing in mind the assumptions underlying this noise model, an SNR of about $0.4(-8 \mathrm{~dB})$.

\section{REFERENCES}

[1] G.S. Brindley, The first 500 patients with sacral anterior root stimulator implants: general description, Paraplegia, 1994, 32, 795805.

[2] N. Granger, D. Chew et al, A microchannel neuroprosthesis for bladder control after spinal cord injury in rat, Science Trans. Med., 5, 210ra155 (2013).

[3] J. Taylor, J. Winter and N. Donaldson, The use of multiple-electrode nerve cuffs for low velocity and velocity-selective neural recording. Med. \& Biol. Eng. \& Comput., 42 (5), 634-43.

[4] N. Donaldson, J. Taylor, R. Rieger and M. Schuettler, Noise and Selectivity of Velocity-Selective Multi-Electrode Nerve Cuffs, Med. \& Biol. Eng. \& Comput., 46 (10), 1005-1018, 2008.

[5] B. Metcalfe, D. Chew, C. Clarke, N. Donaldson and J. Taylor, A new method for spike extraction using VSR, Journal of Neuroscience Methods 251 (2015) 47-55.

[6] M. Schuettler, N. Donaldson, V. Seetohul, and J. Taylor, Fibreselective recording from the peripheral nerves of frogs using a multielectrode cuff, J Neural Eng. 2013;10:36016.

[7] J. Taylor, M. Schuettler, C. Clarke and N. Donaldson, The theory of velocity selective neural recording: a study based on simulation, Med. Biol. Eng. Comput. 50, 309-18, 2012. 